\title{
Stability of time-modulated electroosmotic flow
}

\author{
V. Suresh \\ Department of Chemical Engineering, Stanford University, California 94305-5025 \\ and Department of Biomedical Engineering, University of Michigan, Ann Arbor, Michigan 48109 \\ G. M. Homsy ${ }^{\text {a) }}$ \\ Department of Mechanical and Environmental Engineering, University of California, Santa Barbara, \\ California 93106
}

(Received 17 July 2003; accepted 10 March 2004; published online 25 May 2004)

\begin{abstract}
We present a linear stability analysis of parallel electroosmotic flow in a slot geometry. A spatially uniform time harmonic electric field is applied to a dilute electrolyte solution contained between two infinite parallel plates. The base state ion concentrations and electric potential are determined using the Poisson-Boltzmann equation in the Debye-Hückel approximation. The base velocity field is found to be time harmonic and parallel. It is shown that the original system can be replaced by an equivalent one consisting of an electrically neutral fluid enclosed between oscillating parallel plates, whose speed and frequency of oscillation depend on the modulated electric field. Further, the system of linearized disturbance equations can be decoupled into two stability problems: The first, called the electrokinetic problem, describes the evolution of disturbance ion concentrations and electric potential and is independent of the disturbance velocity components. The second, called the Stokes layer problem describes an oscillatory Stokes layer forced by an electrical body force. The stability of each system is determined by Floquet analysis of a dynamical system obtained from a truncated Galerkin expansion of the perturbation quantities. Our calculations show the system to be linearly stable over a wide range of parameters, with damping rates that become quite small for certain combinations of Stokes and Reynolds numbers. (c) 2004 American Institute of Physics.
\end{abstract}

[DOI: $10.1063 / 1.1736677$ ]

\section{INTRODUCTION AND PROBLEM DEFINITION}

Electroosmosis refers to the bulk motion of a fluid containing charged species under the influence of an applied electric field. While this phenomenon has been known for almost 300 years (see, for e.g., the historical review in Ref. $1)$, recent advances in the fabrication and use of microdevices for the manipulation, separation, and reaction of small quantities of fluids has renewed interest in electroosmosis as a means of transporting fluids in microgeometries. It is therefore important to characterize the stability of such flows. For instance, it may be possible to use instabilities of timemodulated electroosmotic flows to induce micromixing. On the other hand, instabilities may be deleterious to the operation of analytically based "lab-on-a-chip" devices.

There have been few experiments involving ac electroosmotic flows. Instabilities in such systems have recently been reported in a rectangular borosilicate capillary $40 \mathrm{~mm}$ long, $100 \mu \mathrm{m}$ deep, and $1 \mathrm{~mm}$ wide using deionized water. ${ }^{2}$ A spatially homogeneous, sinusoidally alternating electric field was applied by introducing electrodes into fluid reservoirs at either end of the capillary. The field strength was in the range $25-200 \mathrm{kV} / \mathrm{m}$ at a frequency of $20 \mathrm{~Hz}$. Submicron fluorescent particles were used for flow visualization: Particle trajectories indicated time-periodic parallel flow for field strengths below $100 \mathrm{kV} / \mathrm{m}$, but at higher strengths two-

${ }^{a)}$ Electronic mail: bud@engineering.ucsb.edu and three-dimensional motions were observed, suggesting the onset of a flow instability.

Simple one-dimensional solutions to the problem of time-modulated electro-osmosis have been discussed recently. Dutta and Beskok found that ac electroosmotic flow with sinusoidal fields in a two-dimensional (2D) channel is analogous to oscillatory Stokes flow. ${ }^{3}$ Erickson and Li studied flows with more complex wave forms in a rectangular channel of finite aspect ratio. ${ }^{4}$ Reppert and Morgan examined flows in closed capillaries, ${ }^{5}$ where a time-dependent applied potential difference $\Delta V$ induces a time-dependent pressure difference $\Delta P$ that creates a back flow. They performed experiments for the opposite situation where an applied pressure gradient induces a streaming potential ${ }^{6}$ and compared the $\Delta V / \Delta P$ responses for the two cases.

There is, to our knowledge, no analysis of instabilities in such flows or a discussion of the transport mechanisms that might underlie the physical mechanisms of instability. Our main motivation is to provide such a discussion and analyze the stability of the most basic AC electroosmotic flow, the 2D time modulated channel flow.

Consider the slot geometry sketched in Fig. 1 that consists of a dilute electrolyte solution enclosed between two infinite conducting parallel plates which are separated by a distance $2 d$. A Cartesian coordinate system is defined with its origin at the centerline between the two plates. A double layer $^{7}$ of charge exists in the fluid consisting of an immobilized layer of ions at each solid-liquid interface and a diffuse 


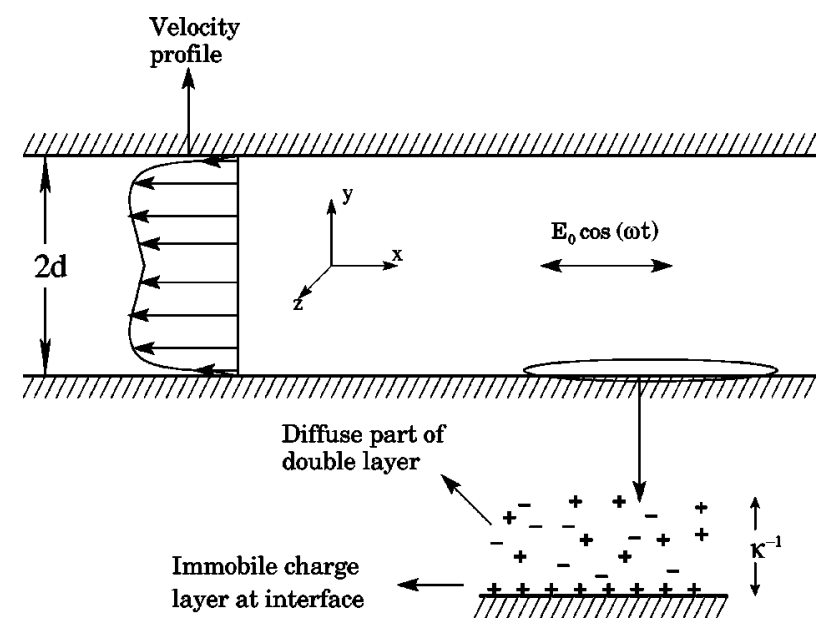

FIG. 1. Flow geometry showing the structure of the double layer and an instantaneous snapshot of the velocity profile.

layer that extends into the bulk, as shown schematically in Fig. 1. Within the double layer the free charge density is not zero and is locally equal to $\rho_{E}^{*}=e \sum_{i=1}^{M} n_{i} c_{i}^{*}$, where $e$ is the charge of an electron, $n_{i}$ is the valence number, $c_{i}^{*}$ the local concentration of species $i$, and $M$ is the total number of charged species in the solution. Associated with this charge distribution is the double layer potential, both of which decay exponentially away from the fluid-solid interface. At large distances from the wall, the solution is therefore electrically neutral, i.e., $\sum_{i=1}^{M} n_{i} c_{i \infty}^{*}=0$, where $c_{i \infty}^{*}$ is the freestream concentration of species $i$. The spatial extent of the double layer is characterized by the Debye length, $\kappa^{-1}$, given $\mathrm{by}^{7}$

$$
\kappa=\left[\frac{N_{A} e^{2}}{\epsilon \epsilon_{0} k_{B} T} \sum_{i=1}^{M} n_{i}^{2} c_{i \infty}^{*}\right]^{1 / 2},
$$

where $N_{A}$ is Avogadro's number, $k_{B}$ is the Boltzmann's constant, $\epsilon_{0}$ is the permittivity of free space, $\epsilon$ is the constant dielectric constant of the bulk fluid, and $T$ is the absolute temperature. $\kappa^{-1}$ is typically small, on the order of a few nanometers.

When a steady axial electric field of amplitude $E_{0}$ is applied to such a system a classical electroosmotic flow is set up. The velocity field is steady and one-dimensional and, outside the Debye layer, nearly an ideal plug flow. Assuming no axial pressure gradient, the velocity scale $U_{0}$ can be estimated by balancing the viscous stress with the electrical body force: The result is $U_{0}=\epsilon \epsilon_{0} k_{B} T E_{0} / \mu e$, where $\mu$ is the constant fluid viscosity of the bulk fluid. Although significant for microfluidic applications, this speed is typically small, of the order of $1 \mathrm{~mm} / \mathrm{s}$, for a dilute aqueous electrolytic solution with a field strength of $100 \mathrm{kV} / \mathrm{m}$.

If instead an AC electric field is applied, the fluid will move back and forth through the slot as the field changes polarity, giving rise to a sloshing motion. The equations admit an exact time-dependent parallel flow which is not necessarily in phase with the applied electric field. In the analysis to follow we determine this base state and study its stability.
The parallel electroosmotic flow is similar to the base state of oscillatory Stokes layers. ${ }^{3}$ Early studies indicated that spatially finite ${ }^{8,9}$ and semi-infinite ${ }^{9}$ Stokes layers are linearly stable, but recent work has shown the latter to be linearly unstable. ${ }^{10}$ However it is unlikely that these instability modes are relevant to microdevices since the largest Reynolds numbers in these devices are at least an order of magnitude smaller than the critical value determined in Ref. 10. In later sections we examine the relation between the electroosmotic flow and the oscillatory Stokes layers, and show that a richer variety of behavior is possible in the current system.

\section{GOVERNING EQUATIONS}

\section{A. Dimensional equations and scaling}

The governing equations arise from conservation equations for the concentration of each charged species, Gauss' law of electrostatics, and the Navier-Stokes equation for the motion of the incompressible bulk fluid. We neglect the effect of gravity and Joule heating and take all transport coefficients to be constant. We start with the dimensional form of the equations in order to make more transparent our choice of scales. Denoting the concentration of species $j$ by $c_{j}^{*}$, its flux by $J_{j}^{*}$, the electric potential by $\Phi^{*}$, the velocity by $\boldsymbol{u}^{*}=\left(u^{*}, v^{*}, w^{*}\right)$, and the pressure by $p^{*}$, the governing equations are

$$
\begin{aligned}
& \frac{\partial c_{j}^{*}}{\partial t}+\boldsymbol{u}^{*} \cdot \boldsymbol{\nabla} c_{j}^{*}=\boldsymbol{\nabla} \cdot \boldsymbol{J}_{j}^{*}=\boldsymbol{\nabla}\left(\varpi_{j} n_{j} c_{j}^{*} e \boldsymbol{\nabla} \Phi^{*}+D_{j} \boldsymbol{\nabla} c_{j}^{*}\right), \\
& \boldsymbol{\epsilon} \epsilon_{0} \nabla^{2} \Phi^{*}+\rho_{E}^{*}=0 \\
& \rho\left(\frac{\partial \boldsymbol{u}^{*}}{\partial t^{*}}+\boldsymbol{u}^{*} \cdot \boldsymbol{\nabla} \boldsymbol{u}^{*}\right)=-\nabla p^{*}+\mu \nabla^{2} \boldsymbol{u}^{*}-\rho_{E}^{*} \boldsymbol{\nabla} \Phi^{*} \\
& \boldsymbol{\nabla} \cdot \boldsymbol{u}^{*}=0
\end{aligned}
$$

$\varpi_{j}$ and $D_{j}$ are the mobility and diffusivity respectively of species $j$, related by the Nernst-Einstein equation

$$
D_{j}=k_{B} T \varpi_{j} .
$$

We see that the total flux of ions, $\boldsymbol{J}_{j}^{*}$, is given by a combination of electrophoresis and gradient diffusion.

Lengths are scaled by the channel half thickness $d$, time by $1 / \omega$, velocities by $U_{0}$, and concentrations by $\sum_{j=1}^{M} n_{j}^{2} c_{j \infty}^{*}$. The choice for the concentration scale arises naturally from the definition of the Debye length in (1). The effective wall potential experienced by the fluid in the diffuse part of the double layer depends on the zeta potential at the solid-fluid interface, which is typically about $0.1 \mathrm{~V}$, i.e., on the order of $k_{B} T / e$ at room temperature. ${ }^{7}$ Thus the electric potential is scaled by $k_{B} T / e$, which normalizes the electrophoretic and diffusive fluxes that appear in (2). Pressure is made dimensionless with the viscous scale $\mu U_{0} / d$ appropriate to slow flows. Dropping the stars denoting the dimensional quantities, the nondimensional governing equations are

$$
\mathrm{Sc}_{j} \mathrm{St} \frac{\partial c_{j}}{\partial t}+\mathrm{Sc}_{j} \operatorname{Re} \boldsymbol{u} \cdot \boldsymbol{\nabla} c_{j}=\boldsymbol{\nabla} \cdot\left(n_{j} c_{j} \nabla \Phi\right)+\nabla^{2} c_{j}
$$




$$
\begin{aligned}
& \nabla^{2} \Phi+\beta^{2} \rho_{E}=0, \\
& \operatorname{St} \frac{\partial \boldsymbol{u}}{\partial t}+\operatorname{Re} \boldsymbol{u} \cdot \boldsymbol{\nabla} \boldsymbol{u}=-\boldsymbol{\nabla} p+\nabla^{2} \boldsymbol{u}-\frac{\beta^{2}}{\gamma_{0}} \rho_{E} \boldsymbol{\nabla} \Phi, \\
& \boldsymbol{\nabla} \times \boldsymbol{u}=0,
\end{aligned}
$$

where $\rho_{E}=\sum_{j}^{M} n_{j} c_{j}$. The various dimensionless parameters are defined as

$\beta=d \kappa$, dimensionless inverse double layer thickness,

$\mathrm{St}=\frac{\omega d^{2}}{\nu}$, Stokes number,

$\mathrm{Re}=\frac{U_{0} d}{\nu}$, electrophoretic Reynolds number,

$\mathrm{Sc}_{j}=\frac{\nu}{D_{j}}$, Schmidt number,

$\gamma_{0}=\frac{E_{0} d e}{k_{B} T}$, dimensionless axial field strength.

Possible sources of instability can be identified as the nonlinear concentration flux terms, $\boldsymbol{u} \cdot \boldsymbol{\nabla} c_{j}$ and $\boldsymbol{\nabla} \cdot\left(n_{j} c_{j} \boldsymbol{\nabla} \Phi\right)$ in (7), as well as the inertial term, $\boldsymbol{u} \cdot \boldsymbol{\nabla} \boldsymbol{u}$, and the electrical forcing term, $\rho_{E} \boldsymbol{\nabla} \Phi$, in (9). We examine these terms in greater detail in Sec. III.

The dimensionless potential $\Phi(x, y, z, t)$ can be decomposed into two parts, one corresponding to the time harmonic axial field and the other to the double layer potential. Thus

$$
\Phi(x, y, z, t)=-\gamma_{0} x \cos (t)+\phi(y, z) .
$$

No slip, no penetration, and no flux conditions are applied at the wall and the zeta potential is fixed there. The flow variables are symmetric about the center of the slot. Therefore we restrict attention to the upper half of the slot $(0 \leqslant y \leqslant 1)$ and specify symmetry conditions on the double layer potential and the velocity components at $y=0$. Anticipating that $\kappa^{-1} \ll d(\beta \gg 1)$, the free-stream concentration of each ionic species is specified at $y=0$. Thus the complete set of boundary conditions is

$$
\begin{aligned}
& \frac{\partial u}{\partial y}=v=\frac{\partial w}{\partial y}=\frac{\partial \phi}{\partial y}=0 \text { at } y=0, \\
& c_{j}=c_{j \infty} \text { at } y=0, \\
& u=v=w=0 \text { at } y=1, \\
& \hat{\boldsymbol{n}} \cdot \boldsymbol{J}_{j}=\frac{\partial c_{j}}{\partial y}+n_{j} c_{j} \frac{\partial \phi}{\partial y}=0 \text { at } y=1, \\
& \phi=1 \text { at } y=1 .
\end{aligned}
$$

At this point it is appropriate to discuss the magnitudes of the various dimensionless parameters, which allows some simplifications in the analysis. Table I lists typical values of the dimensionless parameters for channels that are 50-200 $\mu \mathrm{m}$ thick, binary 1-1 electrolytes at millimolar concentrations at room temperature, electric field strengths of a few kilovolts/ centimeters, and modulation frequencies of $10-50 \mathrm{~Hz}$. Note
TABLE I. Typical values of dimensionless groups for parameter values given in the text.

\begin{tabular}{lc}
\hline \hline$\beta$ & $10^{3}-10^{4}$ \\
$\gamma_{0}$ & 100 \\
$\operatorname{Re}$ & $10^{-1}-1$ \\
$S t$ & $10^{-1}-1$ \\
$S c_{i}$ & $10^{2}-10^{3}$ \\
\hline \hline
\end{tabular}

that $\beta$ is very large even for these thin channels. This enables us to make certain approximations in later sections that significantly reduce the complexity of the stability analysis. $\gamma_{0}$ is large indicating that the applied potential is typically large compared to the zeta potential. Also note that $\mathrm{St}$ and Re are small indicating that inertial effects are small in the momentum equation (9). However the products $\mathrm{Sc}_{j} \mathrm{St}$ and $\mathrm{Pe}_{j}$ $=\mathrm{Sc}_{j} \mathrm{Re}$, where $\mathrm{Pe}_{j}$ is the Peclet number for species $j$, are not small, indicating that convective effects can be important in the species conservation equation (7).

\section{B. Base state}

The governing equations (7)-(10) and the boundary conditions (17)-(21) admit exact solutions of the form $c_{j}$ $=C_{B j}(y), \phi=\phi_{B}(y), \boldsymbol{u}=\left[U_{B}(y, t), 0,0\right]$, i.e., a classical double layer and a parallel flow. In the rest of this work we restrict attention to symmetrical 1-1 electrolytes that consist of two singly charged species, i.e., $M=2, n_{1}=1, n_{2}=-1$, although other cases may be easily studied. The base state is

$$
\begin{aligned}
C_{B j}= & \frac{1}{2} \exp \left[-n_{j} \phi_{B}(y)\right], \\
\phi_{B}(y)= & \frac{\cosh (\beta y)}{\cosh (\beta)}, \\
U_{B}(y, t)= & \operatorname{real}\left[\frac { \beta ^ { 2 } } { \alpha ^ { 2 } - \beta ^ { 2 } } \left(\frac{\cosh (\alpha y)}{\cosh (\alpha)}\right.\right. \\
& \left.\left.-\frac{\cosh (\beta y)}{\cosh (\beta)}\right) \exp (i t)\right],
\end{aligned}
$$

where $\alpha=\sqrt{i \text { St }}$ with $i=\sqrt{-1}$. Equation (22) is the PoissonBoltzmann concentration distribution of the classical double layer. Note that the concentrations are independent of the $x$ component of the electric field and hence steady. $\phi_{B}$ and $U_{B}$ are determined using the Debye-Hückel approximation. ${ }^{7}$ The velocity profile (24) exhibits shearing both inside and outside the double layer with a phase lag for finite Stokes numbers, i.e., finite $\alpha$. For steady fields or slow modulation such that $\alpha$ is small, (24) yields the usual expression for an electroosmotically driven uniform plug flow, plus boundary layer corrections near the walls on the scale of $O(1 / \beta)$.

\section{Approximate base states}

It is clear from (23)-(24) that the base state quantities exhibit boundary layer behavior with terms that decay exponentially fast away from the wall. Since $\beta \gg 1$ we focus at- 
tention on the core region by using approximate forms of the base state valid away from the walls. In the limit $\beta \rightarrow \infty, \alpha$ fixed and for $y \ll 1(22-24)$ reduce to

$$
\begin{aligned}
& C_{B j}(y)=\frac{1}{2}, \\
& \phi_{B}(y)=0, \\
& U_{B}(y, t)=\operatorname{real}\left[-\frac{\cosh (\alpha y)}{\cosh (\alpha)} e^{i t}\right] .
\end{aligned}
$$

Clearly (25)-(26) reflect the bulk electroneutrality of the fluid outside the double layer. Equation (27) is the time dependent counterpart of the Helmholtz-Smoluchowski slip relation ${ }^{7}$ used to model steady electroosmotic flow (which, as noted above, can be recovered in the limit $\mathrm{St} \rightarrow 0$, i.e., $\alpha$ $\rightarrow 0$ ). The flow field defined by (27) is identical to that created by an oscillating wall located at $y=1$. Fluid inertia, represented by $\alpha$, causes the velocity to be out of phase with the wall oscillation. Although the flow field given by (27) is similar to the oscillatory Stokes layer discussed in Ref. 8, there are certain important differences between that work and the problem considered here. Only inertial instabilities are possible in the oscillatory Stokes layer. As we will see in the following section, the coupling of the base velocity and axial electric field to disturbances in the ion concentrations provide other potential instability mechanisms.

\section{LINEAR DISTURBANCE EQUATIONS}

The governing equations (7)-(10) are linearized about the base states given by (26) and (27). We introduce normal mode disturbances of the form

$$
\begin{aligned}
& c_{j}^{\prime}(x, y, z, t)=c_{j}(y, t) \quad e^{i k_{x} x+i k_{z} z}, \\
& \phi^{\prime}(x, y, z, t)=\phi(y, t) \quad e^{i k_{x} x+i k_{z} z}, \\
& \boldsymbol{u}^{\prime}(x, y, z, t)=\boldsymbol{u}(y, t) \quad e^{i k_{x} x+i k_{z} z}, \\
& p^{\prime}(x, y, z, t)=p(y, t) \quad e^{i k_{x} x+i k_{z} z},
\end{aligned}
$$

where we take the wave-vector $\boldsymbol{k}$ to be real. Thus we focus on absolute instabilities. While the issues of convective and absolute instabilities are well understood for steady flows, they are less so for time-dependent flows: see Brevdo and Bridges $^{11}$ for discussion and results on a model problem. The disturbance equations take the form

$$
\begin{aligned}
& \mathrm{Sc}_{j} \mathrm{St} \frac{\partial c_{j}}{\partial t}+i k_{x}\left[\operatorname{Sc}_{j} \operatorname{Re} U_{B}(y, t)+n_{j} \gamma_{0} \cos (t)\right] c_{j}-\frac{\mathcal{L} \phi}{2} \\
& =\mathcal{L} c_{j}, \\
& \mathcal{L} \phi+\beta^{2} \sum_{j=1}^{2} n_{j} c_{j}=0, \\
& \mathrm{St} \frac{\partial u}{\partial t}+\operatorname{Re}\left(i k_{x} U_{B} u+\frac{\partial U_{B}}{\partial y} v\right) \\
& =-i k_{x} p+\mathcal{L} u+\beta^{2} \sum_{j=1}^{2} n_{j} c_{j} \cos (t),
\end{aligned}
$$

$$
\begin{aligned}
& \text { St } \frac{\partial v}{\partial t}+i k_{x} \operatorname{Re} U_{B} v=-\frac{\partial p}{\partial y}+\mathcal{L} v, \\
& \operatorname{St} \frac{\partial w}{\partial t}+i k_{x} \operatorname{Re} U_{B} w=-i k_{z} p+\mathcal{L} w, \\
& i k_{x} u+\frac{\partial v}{\partial y}+i k_{z} w=0,
\end{aligned}
$$

where $(u, v, w)=\boldsymbol{u}$ and $\mathcal{L} \equiv-k_{x}^{2}-k_{z}^{2}+\partial^{2} / \partial y^{2}$. The associated homogeneous boundary conditions are

$$
\begin{aligned}
& \frac{\partial \phi}{\partial y}=\frac{\partial c_{j}}{\partial y}=\frac{\partial u}{\partial y}=v=\frac{\partial w}{\partial y}=0 \text { at } y=0, \\
& \phi=\frac{\partial c_{j}}{\partial y}+\frac{n_{j}}{2} \frac{\partial \phi}{\partial y}=u=v=w=0 \text { at } y=1 .
\end{aligned}
$$

\section{A. Decoupling: Electrokinetic and Stokes layer problems}

Use of the approximate base states allows us to decouple the linear stability equations into two stability problems, which we call the electrokinetic problem and the Stokes layer problem. First note that the term $\boldsymbol{u} \cdot \boldsymbol{\nabla} c_{j}$ in (7) contributes the linear term $\boldsymbol{U}_{B} \cdot \boldsymbol{\nabla} c_{j}^{\prime}$. Therefore there is only one-way coupling between the base flow and the ion fluctuations. As a result the disturbance concentrations and electric potential can be determined solely from the system (32) and (33) and the associated boundary conditions. Growing modes of this system would indicate the presence of instabilities resulting from nonlinearities associated with the convective and electrophoretic fluxes, i.e., the second and third terms in (7). We call this system the electrokinetic problem. Note the coupling of the disturbance concentrations and potential through the term $\mathcal{L} \phi$ in (32) and (33). The phase relationship between the disturbance quantities is complicated: Each species has its own charge relaxation time determined by $\mathrm{Sc}_{j} \mathrm{St}$. For nonzero wave numbers there is a contribution to the phase from the convective transport by the time-periodic base flow $U_{B}(y, t)$ and the applied electric field $\gamma_{0} \cos (t)$ embodied in the second term of (32). While it is not possible to make precise statements regarding the phase relationship, the presence of coupling and forcing terms from the base flow give rise to the possibility of instabilities.

Equations (34) -(37) with the associated boundary conditions define a hydrodynamic stability problem similar to the one obtained for oscillatory Stokes layers. ${ }^{8}$ The last term in (34) is a forcing term from the disturbance electrical body force that can potentially excite modes of the flow problem. We call this system the Stokes layer problem.

\section{B. Galerkin expansion and solution procedure}

We use a Galerkin approximation to treat both the electrokinetic and Stokes layer problems in a manner similar to our previous work. ${ }^{12}$ A general disturbance quantity, $f$ is represented by a truncated Galerkin expansion as $f(y, t)$ $=\sum_{m=0}^{N} A_{m}(t) g_{m}(y)$. The trial functions $g_{m}(y)$ are chosen to satisfy the boundary conditions (38) and (39). Following the 
usual Galerkin procedure, the linear disturbance equations are reduced to a dynamical system of ordinary differential equations

$$
\frac{d \boldsymbol{x}}{d \tau}=2 \pi\left[\boldsymbol{P}+\boldsymbol{Q}_{1} \cos (2 \pi \tau)+\boldsymbol{Q}_{2} \sin (2 \pi \tau)\right] \cdot \boldsymbol{x},
$$

where $\boldsymbol{x}$ is a vector of the time-dependent Galerkin coefficients and $\boldsymbol{P}, \boldsymbol{Q}_{1}$, and $\boldsymbol{Q}_{2}$ are complex valued matrices. Details of the trial functions, inner products and matrices are given in Ref. 13.

The dynamical systems for both the concentration and Stokes layer problems are integrated numerically using the Bulirsch-Stoer method with adaptive time-stepping. ${ }^{14}$ The Floquet exponents were determined by integrating the each system over one time period of modulation starting with the identity matrix as the initial condition. In what follows, we focus on the largest Floquet exponent $\mu_{1}$, with $\mu_{1}<0$ indicating stability. Computations were deemed to have converged when doubling the number of Galerkin modes resulted in the absolute value $\mu_{1}$ changing by less than $\epsilon$ which is defined as

$$
\epsilon= \begin{cases}10^{-3} & \text { if }\left|\mu_{1}\right| \geqslant 10^{-2} \\ 0.1\left|\mu_{1}\right| & \text { if }\left|\mu_{1}\right|<10^{-2} .\end{cases}
$$

Typically, 16 to 128 modes were required for the electrokinetic problem and 8 to 64 modes for the Stokes layer problem. Below, we present results for each problem separately.

\section{RESULTS}

We fix $\mathrm{Sc}_{1}=750, \mathrm{Sc}_{2}=492$ corresponding to the values for sodium and chloride ions at $T=298 \mathrm{~K}$. We then fix $\beta$ 5269, which corresponds to $d=50 \mu \mathrm{m}, c_{j, \infty}^{*}=10^{-3} \mathrm{M}$. The dynamical system is solved for values of the other parameters in the range $50 \leqslant \gamma_{0} \leqslant 500,0 \leqslant \mathrm{Re} \leqslant 20,0.25 \leqslant \mathrm{St}$ $\leqslant 20$. With reference to Table I, this wide range covers most microfluidic applications.

\section{A. The electrokinetic problem}

As a validation, the static double layer was found to be stable, as expected. In the case of modulated electric field $\left(\gamma_{0} \neq 0\right)$ the leading Floquet exponent was found to be real in the parameter range studied. A Squire's transformation does not exist for this problem. However since $k_{z}$ only appears in the diffusive terms, modes with $k_{z} \neq 0$ are likely to be more stable (less unstable) than 2D modes: Accordingly, we consider two-dimensional disturbances by setting $k_{z}=0$.

In the long wave limit $k_{x}=0$ the linear stability equations can be cast in the form of an eigenvalue problem which only admits solutions of the form $c_{1}=c_{2}=$ constant, $\phi$ $=0$ with a zero growth rate. Note that $c_{1}$ and $c_{2}$ can only be determined within a constant because of the derivative boundary conditions and thus $k_{x}=0$ is a trivial case of neutral stability.

Figures 2(a)-2(d) show the leading Floquet exponent $\mu_{1}$ as a function of $k_{x}$ for $\gamma_{0}=100$ and different values of $\mathrm{Re}$ and St. St is held fixed in Figs. 2(a) and 2(b) while Re is held fixed in Figs. 2(c) and 2(d). $\mu_{1}$ is found to be negative,

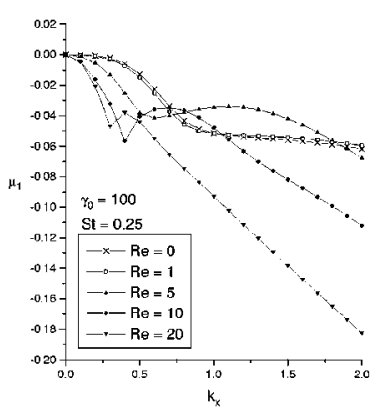

(a)

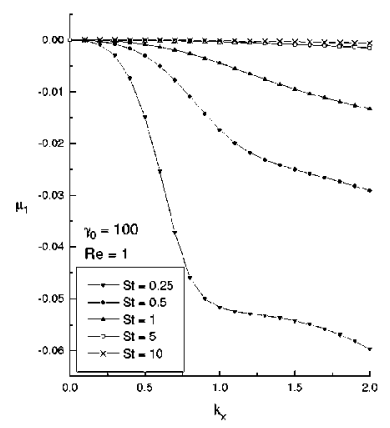

(c)

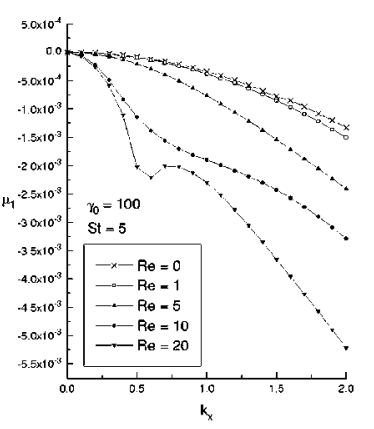

(b)

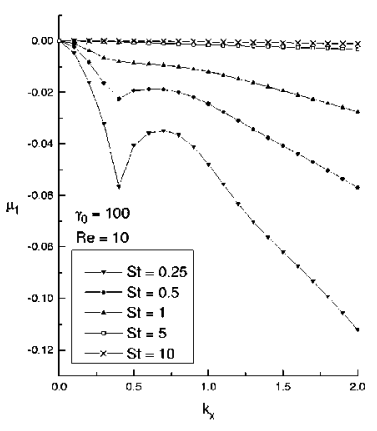

(d)
FIG. 2. Leading Floquet exponent $\mu_{1}$ vs $k_{x}$ (a) $\gamma_{0}=100, \mathrm{St}=0.25$, (b) $\gamma_{0}$ $=100, \mathrm{St}=5$, (c) $\gamma_{0}=100, \mathrm{Re}=1$, and (d) $\gamma_{0}=100, \mathrm{Re}=10$.

indicating stability, and is typically small, $O\left(10^{-3}-10^{-2}\right)$. As $k_{x}$ increases $\mu_{1}$, in general, becomes increasingly negative, indicating increasing degrees of stability. However local maxima are observed for certain combinations of St and Re. For a given St these maxima occur when Re exceeds a minimum value, which increases with St [Figs. 2(a) and 2(b)]. Curves for different Re cross at small St [Fig. 2(a)] while $\mu_{1}$ decreases monotonically with $\mathrm{Re}$ at larger St [Fig. 2(b)]. Figs. 2(c) and 2(d) indicate that $\mu_{1}$ increases with St for all wave numbers and becomes quite close to zero for $\mathrm{St} \geqslant 5$. Figure 3 examines in greater detail the Stokes number dependence of $\mu_{1}$ at different wave numbers for fixed $\gamma_{0}$ Re. $\mu_{1}$ increases rapidly with St and asymptotically approaches zero without becoming positive. Similar results were obtained for other parameter values. While these figures do not indicate any instability, interesting crossovers are seen in the wave number range $0.5-1.5$. We therefore examine the system behavior by varying either $\gamma_{0}$ or Re while holding the other fixed for $k_{x}=0.5,1.0,1.5$. Graphs are plotted for $\mathrm{St}$ $=0.25,5$ to compare results at small and large St.

Figures 4(a)-4(d) show the effect of varying Re at fixed $\gamma_{0}$, St on $\mu_{1}$. At $\mathrm{St}=0.25$ [Figs. 4(a)-4(b)] the system initially becomes more stable as $\mathrm{Re}$ is increased for $k_{x}=0.5$. When $\mathrm{Re}$ is further increased $\mu_{1}$ passes through a maximum in the range $10 \leqslant \operatorname{Re} \leqslant 15$. For $k_{x}=1,1.5$ the system becomes less stable as $\mathrm{Re}$ is increased from 0 and $\mu_{1}$ passes through a maximum in the range $5 \leqslant \mathrm{Re} \leqslant 10$. At small $\mathrm{Re}$ the relative stability of the three wave numbers depends on $\gamma_{0}: k_{x}$ $=0.5$ is less stable than $k_{x}=1.5$ at $\gamma_{0}=100$, but more stable at $\gamma_{0}=400$. For $\operatorname{Re}>12.5 k_{x}=0.5$ is the least stable of the three for both $\gamma_{0}=100,400$. For $\mathrm{St}=5$ [Figs. 4(c) and $4(\mathrm{~d})$ ] 


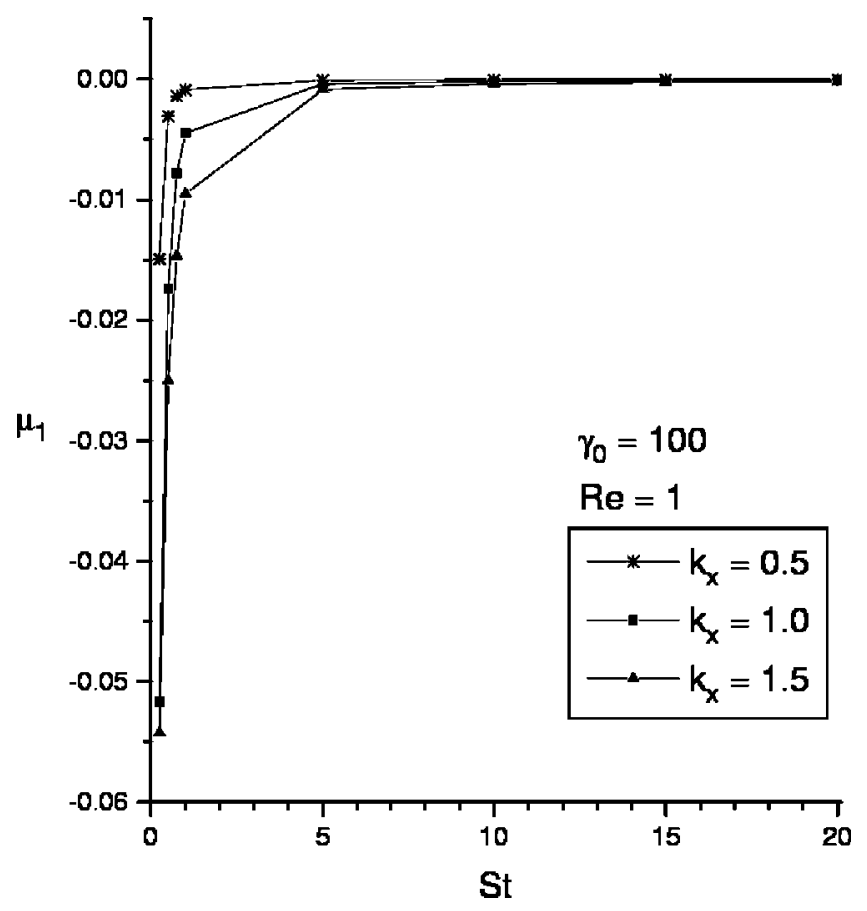

FIG. 3. Leading Floquet exponent $\mu_{1}$ versus St for $k_{x}=0.5,1.0,1.5$; Re $=1 ;$ and $\gamma_{0}=100$.

curves for different wave numbers do not cross. $\mu_{1}$ decreases monotonically with Re at $\gamma_{0}=100$, but local minima and maxima are seen at $\gamma_{0}=400$. Also, the curves for smaller wave numbers are less stable, irrespective of Re and $\gamma_{0}$.

Figures 5(a) -5 (d) show a similar parametric study of $\mu_{1}$

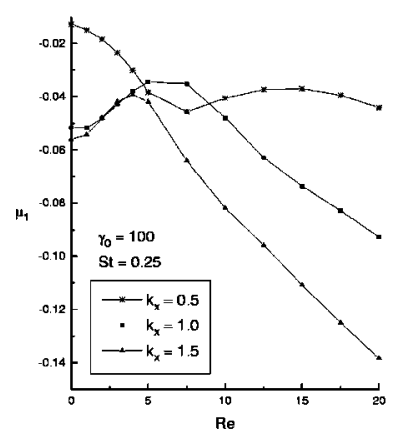

(a)

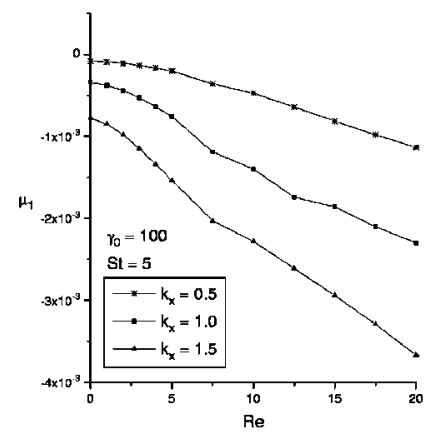

(c)

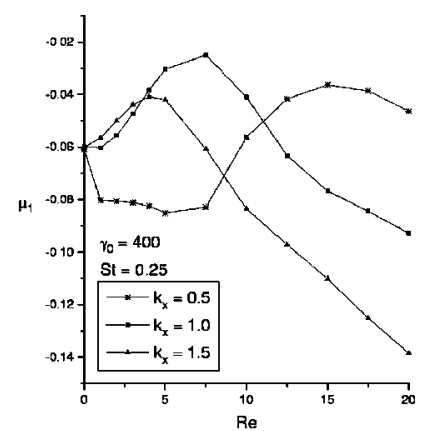

(b)

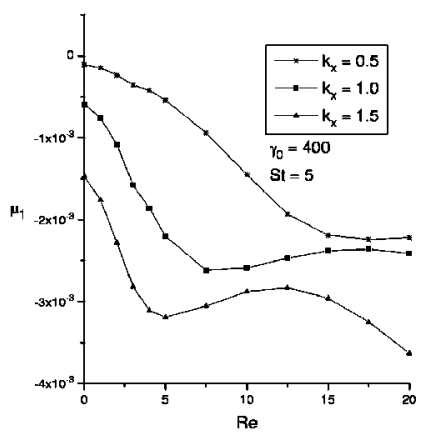

(d)
FIG. 4. Leading Floquet exponent $\mu_{1}$ vs $\operatorname{Re}(a) \gamma_{0}=100, \mathrm{St}=0.25$, (b) $\gamma_{0}$ $=400, \mathrm{St}=0.25$, (c) $\gamma_{0}=100, \mathrm{St}=5$, and (d) $\gamma_{0}=400, \mathrm{St}=5$.

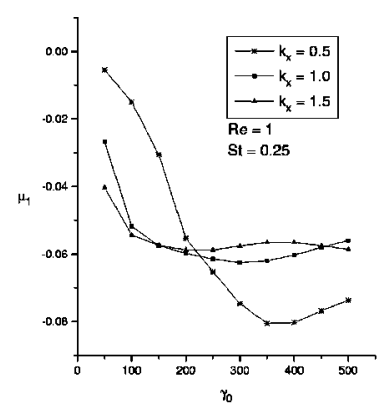

(a)

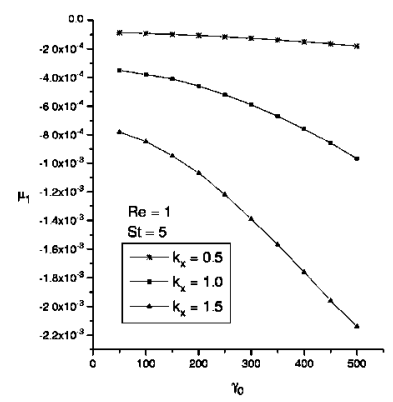

(c)

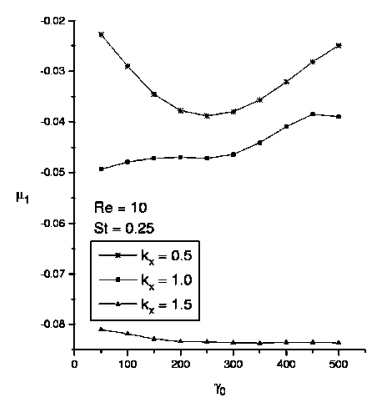

(b)

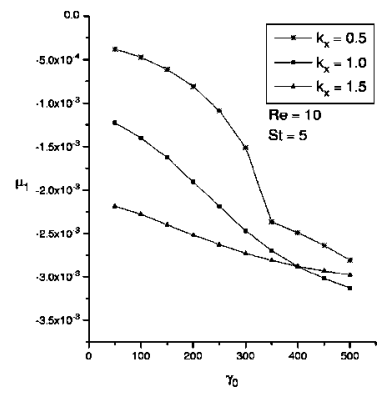

(d)
FIG. 5. Leading Floquet exponent $\mu_{1}$ vs $\gamma_{0}$ (a) $\mathrm{Re}=1, \mathrm{St}=0.25$, (b) $\mathrm{Re}$ $=10, \mathrm{St}=0.25$, (c) $\mathrm{Re}=1, \mathrm{St}=5$, and (d) $\mathrm{Re}=10, \mathrm{St}=5$.

as a function of $\gamma_{0}$ at fixed Re, St. When $\mathrm{St}=0.25, \mu_{1}$ initially decreases as $\gamma_{0}$ is increased [Figs. 5(a) and 5(b)], except for $k_{x}=1, \operatorname{Re}=10$. At higher values of $\gamma_{0}$ the curves pass through local minima and/or maxima. The behavior of the $k_{x}=0.5, \mathrm{Re}=10$ curve [Fig. 5(b) ]is particularly interesting as it seems to suggest instability with increasing $\gamma_{0}$. Computations in the range $500 \leqslant \gamma_{0} \leqslant 2000$ (not plotted) showed that $\mu_{1}$ attains a maximum value of about -0.015 at $\gamma_{0}=1000$ and then decreases. It seems likely that the curves for $k_{x}=1,1.5$ at $\operatorname{Re}=1$, which increase when $\gamma_{0}>400$, behave similarly. For $\mathrm{St}=5$ (Figs. 5(c) and 5(d)) $\mu_{1}$ decreases monotonically with $\gamma_{0}$.

These results show that the dependence of $\mu_{1}$ on the various parameters is complex. However, there are a few generalizations that may be made as follows:

(1) Linear theory does not predict any purely electrokinetic instabilities over the range of parameters studied. In addition there is a likelihood, based on extrapolation of the available results, that this system is linearly stable for all values of the parameters.

(2) Small perturbations are damped, but only slightly so, with the damping rate becoming arbitrarily small at large St for particular choices of the other parameters [refer to Fig. (3)].

(3) The reason for the approach of $\mu_{1}$ to zero at large St is not simple to discern. Stability or instability is determined by the linearization of the nonlinear terms in the transport equation (7). These result in the final three terms on the left-hand side of (32). The parametric studies show that increasing $\gamma_{0}$ at small Re results in damping. On the other hand, increasing St has two effects: Increasing the degree of shearing of the base state via 
(27) and increasing the charge relaxation time via (32). The approach to neutral stability is probably due to a complicated relationship between the convection of charge by the base state velocity at finite $\left(k_{x}, \mathrm{Re}\right)$ and charge relaxation.

\section{B. Stokes layer problem}

A comprehensive study of the stability of spatially finite oscillatory Stokes layers was first performed by Kerczek and Davis, ${ }^{8}$ who showed that such layers are linearly stable over a wide parameter range. Hall $^{9}$ used a different approach to study semi-infinite layers, but also confirmed the stability of finite layers. Recently, the semi-infinite Stokes layer has been shown to be linearly unstable ${ }^{11}$ with a critical Reynolds number $\operatorname{Re}_{\delta} \approx 1400$ based on the Stokes layer thickness, $\delta$ $=\sqrt{2 \nu / \omega}$. We do not expect these instability modes to be active at the smaller Reynolds numbers $\left(\operatorname{Re}_{\delta}=\sqrt{2 / \mathrm{St}} \mathrm{Re}\right.$ $<60$ ) of our system. We compare our results with Kerczek and Davis since our approach closely parallels theirs. The current problem differs from that work in certain respects.

In contrast to the unforced system of Ref. 8, the Stokes layer here is forced by the electrical body force term in (34). However, since the electrokinetic problem is damped, no persistent forcing exists for the flow equations. Therefore it is sufficient to consider the stability of the unforced Stokes layer.

The boundary conditions and the parameter range considered here are also different. We apply a symmetry condition at the center of the slot $(y=0)$ in contrast to the no-slip condition at the stationary upper solid boundary used by Kerczek and Davis. Their work used a quantity $\beta$, which we will refer to as $\chi$ to avoid confusion with the parameter $\beta$ used here. In terms of the scales used in this work $\chi=\sqrt{2 \mathrm{St}}$. Kerczek and Davis used $\chi=8$, corresponding to $\mathrm{St}=32$ and Reynolds numbers $100 \leqslant \operatorname{Re}_{\delta} \leqslant 800$. Thus both the Reynolds and Stokes numbers used here are up to one order of magnitude smaller.

Kerczek and Davis found the oscillatory Stokes layer to be linearly stable throughout the parameter range studied. We did not expect this conclusion to be altered by the slightly different boundary conditions used here. This was borne out by our calculations (not shown) which demonstrated that the disturbances were highly damped. ${ }^{13}$ It is clear that the Stokes and Reynolds numbers are too small to excite shear instabilities of the system.

\section{SUMMARY}

We have performed a linear stability analysis of electroosmotic flow in a slot geometry. The thinness of the electrical double layer allowed the use of particularly simple forms of the base states valid in the central portion of the slot away from the solid boundaries, which in turn led to the decoupling of the linear stability equations into an electrokinetic problem and a Stokes layer problem.

While the parallel flow base states are very closely related to the base states of oscillatory Stokes layers, different instability mechanisms are possible in modulated electroosmotic flow. These mechanisms are seen in the electroki- netic problem through the coupling of disturbance concentration and electric potential fields to the base state quantities, including convective coupling since Peclet numbers are $O(1)$. Neutral stability is trivially achieved in the long wave limit. No instabilities of the electrokinetic problem were found at nonzero wave numbers in the parameter range studied, although the damping rates are typically small and can, for some ranges of parameters, become arbitrarily close to zero.

The Stokes layer problem was found to be highly damped, which is consistent with previous linear stability results for spatially finite Stokes layers, ${ }^{8,9}$ even though such flows have been observed to be unstable in experiments. Recent linear stability results have shown the semi-infinite Stokes layer to be unstable, ${ }^{11}$ but the critical Reynolds number for instability is at least an order of magnitude higher than those relevant in microfluidic applications.

We can draw some important conclusions from these results. Our analysis shows that, away from solid boundaries, it is possible to decouple electrokinetic effects due to the convective and electrophoretic flux of charge from purely hydrodynamic effects in the linear stability equations. The results also indicate that the electrokinetic problem is less stable than the Stokes layer problem in the parameter regime relevant to microdevices.

It remains to comment on the relationship between our theory and the experiments in Ref. 2. The Floquet theory used here adopts a particular definition of asymptotic stability, i.e., an absolute instability with no net growth over one cycle. We do not consider the possibility of convective instabilities. Our analysis uses the Debye-Hückel and thin double layer approximations to obtain the base state. It is unlikely that these approximations affect the stability results since the neglected terms decay exponentially fast away from the boundary. It is conceivable that the observed instabilities are due to transient growth over part of the cycle leading to nonlinear transition, or convective instabilities associated with spatial growth. It is also possible that the instabilities seen in experiments result from fundamentally finite amplitude disturbances and cannot be predicted by linear analysis. Such subcritical instabilities would probably result from the weakly damped electrokinetic modes rather than the shear associated with the Stokes layer. Finally, instabilities may be due to mechanisms such as Joule heating that are not considered here. In any event, the results presented here constitute a case against which to evaluate these other effects.

\section{ACKNOWLEDGMENTS}

Primary support for this work was through a grant from the Microgravity Science Division of NASA (Grant No. NAG3-1943). V.S. gratefully acknowledges support from the Stanford Graduate Fellowship Program. The authors would like to thank the reviewers whose suggestions helped improve an earlier version of the article.

\footnotetext{
${ }^{1}$ S. S. Dukhin, "Development of notions as to the mechanism of electrokinetic phenomena and the structure of the colloid micelle," in Surface and Colloid Science, edited by E. Matijević (Wiley, New York 1974), Vol. 7 , pp. 2-47.
} 
${ }^{2}$ M. H. Oddy, J. D. Santiago, and J. C. Mikkelsen, "Electrokinetic instability micromixing," Anal. Chem. 73, 5822 (2001).

${ }^{3}$ P. Dutta and A. Beskok, "Analytical solution of time periodic electroosmotic flows: Analogies to Stokes' second problem," Anal. Chem. 73, 5097 (2001).

${ }^{4} \mathrm{D}$. Erickson and D. Li, "Analysis of alternating current electroosmotic flows in a rectangular microchannel," Langmuir 19, 5421 (2003).

${ }^{5}$ P. M. Reppert and F. D. Morgan, "Frequency-dependent electroosmosis," J. Colloid Interface Sci. 254, 372 (2002).

${ }^{6}$ P. M. Reppert, F. D. Morgan, D. P. Lesmes, and L. Jouniauxz, "Frequency-dependent streaming potentials," J. Colloid Interface Sci. 234, 194 (2001).

${ }^{7}$ R. F. Probstein, Physicochemical Hydrodynamics (Wiley, New York, 1994).
${ }^{8}$ C. V. Kerczek and S. H. Davis, "Linear stability theory of oscillatory Stokes layers," J. Fluid Mech. 62, 753 (1974).

${ }^{9}$ P. Hall, "The linear stability of flat Stokes layers," Proc. R. Soc. London, Ser. A 359, 151 (1978).

${ }^{10}$ P. J. Blennerhassett and A. P. Bassom, "The linear stability of flat Stokes layers," J. Fluid Mech. 464, 393 (2002).

${ }^{11}$ L. Brevdo and T. J. Bridges, "Absolute and convective instabilities of temporally oscillating flows," Z. Angew. Math. Phys. 48, 290 (1997).

${ }^{12}$ V. Suresh and G. M. Homsy, "Stability of return thermocapillary flows and gravity modulation," Phys. Fluids 13, 3155 (2001).

${ }^{13} \mathrm{~V}$. Suresh, "Stability of flows subject to time-dependent body forces," Ph.D. thesis, Stanford University, California, 2002.

${ }^{14}$ W. H. Press, S. A. Teukolsky, W. T. Vetterling, and B. P. Flannery, Numerical Recipes in Fortran (Cambridge University, Cambridge, England, 1994). 